\title{
The function-indexed sequential empirical process under long-range dependence
}

\author{
JANNIS BUCHSTEINER
}

Fakultät für Mathematik, Ruhr-Universität Bochum, Universitätsstraße 150, 44801 Bochum, Germany. E-mail: jannis.buchsteiner@rub.de

Let $\left(\boldsymbol{X}_{j}\right)_{j \geq 1}$ be a multivariate long-range dependent Gaussian process. We study the asymptotic behavior of the corresponding sequential empirical process indexed by a class of functions. If some entropy condition is satisfied we have weak convergence to a linear combination of Hermite processes.

Keywords: entropy condition; Hermite process; multivariate long-range dependence; sequential empirical process

\section{Introduction}

For a real-valued stationary process $\left(Y_{j}\right)_{j \geq 1}$ the sequential empirical process $\left(R_{N}(x, t)\right)$ is defined by

$$
R_{N}(x, t):=\sum_{j=1}^{\lfloor N t\rfloor}\left(1_{\left\{Y_{j} \leq x\right\}}-P\left(Y_{j} \leq x\right)\right), \quad x \in \mathbb{R}, t \in[0,1] .
$$

This process plays an important role in nonparametric statistics, for example, in change-point analysis. If $\left(Y_{j}\right)_{j \geq 1}$ is a subordinated Gaussian process which exhibits long-range dependence, weak convergence was established by Dehling and Taqqu [9]. The limiting process is given by the product of a deterministic function and an Hermite process $\left(Z_{m}(t)\right)_{0 \leq t \leq 1}$. The latter can be represented as a stochastic integral in the spectral domain, more precisely

$$
Z_{m}(t)=K(m, D) \int_{\mathbb{R}^{m}}^{\prime \prime} \frac{e^{i t\left(x_{1}+\cdots+x_{m}\right)}-1}{i\left(x_{1}+\cdots+x_{m}\right)} \prod_{j=1}^{m}\left|x_{j}\right|^{-(1-D) / 2} \tilde{B}\left(d x_{1}\right) \cdots \tilde{B}\left(d x_{m}\right),
$$

where $\tilde{B}$ is a suitable random spectral measure and $K(m, D)$ is normalizing constant. The double prime indicates that integration excludes not only $\left\{x \in \mathbb{R}^{m}: x^{(i)}=x^{(j)}, 1 \leq i<j \leq m\right\}$ but also $\left\{x \in \mathbb{R}^{m}: x^{(i)}=-x^{(j)}, 1 \leq i<j \leq m\right\}$. For details and further representations, see [22] and [18]. A first step in generalizing this result to multivariate observations was done by Marinucci [16]. He studied the asymptotics of the empirical process $\left(R_{N}(x, 1)\right)$ based on a two-dimensional longrange dependent process, where $\leq$ in (1) is understood componentwise. However, this is nothing but checking if the observation lies inside a rectangle or not. Since there is no reason to restrict ourselves to such specific sets, it could be interesting to study indicators of balls or ellipsoids as well. This purpose yields the sequential empirical process indexed by a class of functions $\mathcal{F}$. 
Definition 1. Let $\left(\boldsymbol{X}_{j}\right)_{j \geq 1}$ be stationary $\mathbb{R}^{p}$-valued process and let $\mathcal{F} \subset L^{2}\left(P_{\boldsymbol{X}_{1}}\right)$ be a class of square-integrable functions. Furthermore, let $\mathcal{F}$ be uniformly bounded, i.e. $\sup _{f \in \mathcal{F}}|f(x)|<\infty$ for all $x \in \mathbb{R}^{p}$. We define the function-indexed sequential empirical process $\left(R_{N}(f, t)\right)_{\mathcal{F} \times[0,1]}$ by

$$
R_{N}(f, t)=\sum_{j=1}^{\lfloor N t\rfloor} f\left(\boldsymbol{X}_{j}\right)-E\left(f\left(\boldsymbol{X}_{1}\right)\right)
$$

Most asymptotic results one can find in the literature are formulated for the one-parameter process $\left(R_{N}(f, 1)\right)$. Under independence Dudley [11] studied the empirical process indexed by a class of measurable sets, that is, he considered $\mathcal{F}=\left\{1_{A}(\cdot): A \in \mathcal{A}\right\}$, where $\mathcal{A}$ is a suitable subset of the Borel $\sigma$-algebra. He stated different assumptions under which weak convergence to a Gaussian process holds, including a so-called metric entropy with inclusion. Generalizing this idea, Ossiander [17] introduced $L^{2}$-brackets to approximate the elements of $\mathcal{F}$. These brackets allow to study larger classes of functions as long as a metric entropy integrability condition is satisfied, see [17], Theorem 3.1. A bracketing condition under strong mixing was stated by Andrews and Pollard [1]. Doukhan, Massart and Rio [10] studied the function-indexed empirical process for $\beta$-mixing sequences. The case of Gaussian long-range dependent random vectors was already handled by Arcones [2], Theorem 9. The assumption on the bracketing number therein is very restrictive and will be considerably improved in this paper. Note, the only known result for the two-parameter process $\left(R_{N}(f, t)\right)$ was given by Dehling, Durieu and Tusche [7] for multiple mixing data.

The simplest way to define multivariate long-range dependence for an $\mathbb{R}^{p}$-valued process $\left(\boldsymbol{X}_{j}\right)_{j \geq 1}, \boldsymbol{X}_{j}=\left(X_{j}^{(1)}, \ldots, X_{j}^{(p)}\right)$, is the following one. Assume that the component-processes $\left(X_{j}^{(1)}\right), \ldots,\left(X_{j}^{(p)}\right)$ are independent and that each of them individually satisfies the well-known time-domain long-range dependence condition that is

$$
r^{(i)}(k)=\operatorname{Cov}\left(X_{1}^{(i)}, X_{1+k}^{(i)}\right)=k^{-D_{i}} L_{i}(k), \quad 1 \leq i \leq p .
$$

For $p=2$, this concept was used by Marinucci [16] and, if $L_{1}=L_{2}$ and $D_{1}=D_{2}$, by Taufer [23]. However, using this approach we can not model dependency between different components. Therefore, we have to replace the assumption of independent components by a suitable crosscovariance structure. For $1 \leq i, j \leq p$ we call the function

$$
r^{(i, j)}(k)=\operatorname{Cov}\left(X_{1}^{(i)}, X_{1+k}^{(j)}\right)
$$

a cross-covariance function. Ho and Sun [13] considered a stationary bivariate Gaussian process such that for $1 \leq i, j \leq 2, r^{(i, j)}(k)$ is asymptotic equal to $k^{-\beta_{i, j}}, \beta_{i, j}>0$. The definition of multivariate long-range dependence we use here can also be labeled as a single-parameter longrange dependence. It was originally stated by Arcones [2], page 2259, see also Beran et al. [4], page 300 , and is included in the very general concept of multivariate long-range dependence given by Kechagias and Pipiras [14], Definition 2.1. 
Definition 2. A stationary $\mathbb{R}^{p}$-valued process $\left(\boldsymbol{X}_{j}\right)_{j \geq 1}$ with finite second moments is called long-range dependent if for each $1 \leq i, j \leq p$

$$
r^{(i, j)}(k)=c_{i j} k^{-D} L(k) \quad \text { for } k \geq 1,
$$

where $L$ is slowly varying at infinity, $0<D<1$, and $c_{i j} \in \mathbb{R}$ are not all equal to zero.

In the definition given in [14] the long-range dependence parameter $D$ also depends on $i$ and $j$ so that the cross-covariance is given by

$$
r^{(i, j)}(k)=c_{i j} k^{-\left(D_{i}+D_{j}\right) / 2} L(k), \quad 0<D_{i}, D_{j}<1 .
$$

Although this assumption is more realistic, multiple different parameters yield a degenerate limiting behavior, see Leonenko, Sakhno and Taufer [15], page 308. We briefly discuss this phenomenon subsequent to Theorem 3 .

\section{Assumptions and techniques}

From the probabilistic point of view, we will interpret $\left(R_{N}(f, t)\right)$ as a random element in $\ell^{\infty}(\mathcal{F} \times[0,1])$, that is the space of real-valued bounded functions on $\mathcal{F} \times[0,1]$. The uniformly boundedness of $\mathcal{F}$ stated in Definition 1 ensures well-definedness. We equip $\ell^{\infty}(\mathcal{F} \times[0,1])$ with the supremum norm and the corresponding Borel $\sigma$-algebra. In general, empirical processes are not measurable with respect to the Borel $\sigma$-algebra generated by the uniform metric, see [5], page 157. Dehling and Taqqu [9] handled this problem by considering the open ball $\sigma$-algebra instead of the Borel one. We will use the idea of outer expectation introduced by HoffmannJørgensen. For the sake of completeness we recall the definition. A detailed introduction to this concept can be found in [24], Chapter 1.

Definition 3. Let $(\Omega, \mathcal{A}, P)$ be an arbitrary probability space and let $\hat{\mathbb{R}}$ be the extended real line equipped with the Borel $\sigma$-algebra.

(i) For any $B \subset \Omega$, we call

$$
P^{*}(B):=\inf \{P(A): A \supset B, A \in \mathcal{A}\}
$$

the outer probability.

(ii) For any map $X: \Omega \rightarrow \hat{\mathbb{R}}$ we call

$$
E^{*} X:=\inf \{E Y: Y \geq X, Y: \Omega \rightarrow \hat{\mathbb{R}} \text { measurable and } E Y \text { exists }\}
$$

the outer integral of $X$.

(iii) A sequence of maps $\left(X_{n}\right)_{n \geq 1}$, taking values in a metric space $S$, converges weakly to a Borel-measurable random variable $X: \Omega \rightarrow S$ if

$$
E^{*} f\left(X_{n}\right) \longrightarrow E f(X)
$$

for all continuous, bounded functions $f: S \rightarrow \mathbb{R}$. 
If measurability holds, part (iii) of the above definition conforms with classical weak convergence of random variables. Moreover, one can show that the Portmanteau theorem, continuous mapping theorem, Prohorov's theorem, and other well-known results are still applicable in the context of outer weak convergence, see [24], Section 1.3. Convergence in outer probability and outer almost sure convergence can be defined analogous.

From now on, assume that the process $\left(\boldsymbol{X}_{j}\right)_{j \geq 1}$ is standard normal and long-range dependent in the sense of Definition 2, that is,

$$
\begin{aligned}
E X_{1}^{(i)} & =0, \quad 1 \leq i \leq p, \\
E X_{1}^{(i)} X_{1}^{(j)} & =\delta_{i j}, \\
r^{(i, j)}(k) & =c_{i j} L(k) k^{-D} \quad \text { for } k \geq 1 .
\end{aligned}
$$

The existence of such a process is ensured because there exist a linear representation for multivariate long-range dependent processes, see [14], Corollary 4.1. Condition (5) is not restrictive, since for an arbitrary covariance matrix $\Sigma$ we have $G \boldsymbol{X}_{1} \sim \mathcal{N}(0, \Sigma)$, where $G$ is the Cholesky decomposition of $\Sigma$. In this case, one can study $\left(R_{N}(f \circ G, t)\right)$ instead of $\left(R_{N}(f, t)\right)$.

By $\mathcal{N}_{p}$ we denote a $p$-dimensional standard normal distribution and by $H_{k}$ the $k$ th Hermite polynomial given by

$$
H_{k}(x):=(-1)^{k} e^{x^{2} / 2} \frac{d^{k}}{d x^{k}} e^{-x^{2} / 2} .
$$

Using these univariate polynomials, we define multivariate Hermite polynomials by

$$
H_{l_{1}, \ldots, l_{p}}(x):=H_{l_{1}}\left(x^{(1)}\right) H_{l_{2}}\left(x^{(2)}\right) \cdots H_{l_{p}}\left(x^{(p)}\right), \quad x \in \mathbb{R}^{p} .
$$

The collection of all multivariate Hermite polynomials of order $p$ forms an orthogonal basis of $L^{2}\left(\mathcal{N}_{p}\right)$, see [4], page 122. Thus, for any $f \in \mathcal{F} \subset L^{2}\left(\mathcal{N}_{p}\right)$ we have a $L^{2}$-series expansion of $f\left(\boldsymbol{X}_{j}\right)-E\left(f\left(\boldsymbol{X}_{1}\right)\right)$ namely

$$
f\left(\boldsymbol{X}_{j}\right)-E\left(f\left(\boldsymbol{X}_{1}\right)\right)=\sum_{k=m(f)}^{\infty} \sum_{l_{1}+\cdots+l_{p}=k} \frac{J_{l_{1}, \ldots, l_{p}}(f)}{l_{1} ! \cdots l_{p} !} H_{l_{1}, \ldots, l_{p}}\left(\boldsymbol{X}_{j}\right) .
$$

The index $m(f) \geq 1$ denotes the order of the first non-zero Hermite coefficient. We call the minimum $m:=\min \{m(f): f \in \mathcal{F}\}$ the Hermite rank of $\mathcal{F}$. Note, the Hermite rank $m$ is only determined by $f$ and not by a specific choice of $c_{i j}$.

Next we must account for the fact that the elements of $\mathcal{F}$ can be well approximated based on a relatively small number of functions. The definition of $L^{2}$-brackets we give next based on that of Ossiander [17].

\section{Definition 4.}

(i) For $\varepsilon>0$ and $l, u \in \mathcal{F}$ with $l \leq u$ we call $\{f \in \mathcal{F}: l \leq f \leq u\}$ a $\varepsilon$-bracket if $\|u-l\|_{2} \leq \varepsilon$, where $\|\cdot\|_{2}$ denotes the $L^{2}$-norm with respect to $\mathcal{N}_{p}$. 
(ii) The smallest number $N(\varepsilon)$ of $\varepsilon$-brackets needed to cover $\mathcal{F}$ is called bracketing number.

Obviously, the bracketing number increases if $\varepsilon$ tends to zero. Therefore, we assume that the following entropy condition holds

$$
\int_{0}^{1} \varepsilon^{r-1} N(\varepsilon)^{2} d \varepsilon<\infty \quad \text { for some integer } r \geq 1 .
$$

Roughly speaking, condition (6) is satisfied as long as $N(\varepsilon)$ grows polynomial in $\varepsilon^{-1}$. Although under independence one can handle exponential growth rates, see [17], Theorem 3.1, the entropy condition we require here is not untypical for the dependent case. Similar conditions were stated in [1], Theorem 2.2, and [7], Theorem 2.5. Besides, (6) is a much weaker assumptions than the one used in [2], (5.1), namely

$$
\int_{0}^{1} N(\varepsilon) d \varepsilon<\infty .
$$

Additionally, we need uniformly bounded $2 q$ th moments for all $f \in \mathcal{F}$, where $q$ depends on the Hermite rank $m$, the long-range dependence parameter $D$, and $r$ from (6). More precisely,

$$
\sup _{f \in \mathcal{F}} E\left(f\left(\boldsymbol{X}_{1}\right)\right)^{2 q}<\infty, \quad \text { where } 2 q>\frac{m D r}{1-m D} \vee m r .
$$

This choice of range for $2 q$ is determined by technical constraints arising in the proof of Lemma 3. The lower bound is $m r$, if and only if $D<1 /(m+1)$.

\section{Results}

Theorem 1. Let $\left(\boldsymbol{X}_{j}\right)_{j \geq 1}$ be a p-dimensional standard Gaussian long-range dependent process in the sense of Definition 2 and let $\mathcal{F}$ be a uniformly bounded class of functions satisfying (6) and (7). Moreover, let $0<D<1 / m$, where $m$ is the Hermite rank of $\mathcal{F}$, and set

$$
d_{N}^{2}=\operatorname{Var}\left(\sum_{j=1}^{N} H_{m}\left(X_{j}^{(1)}\right)\right)
$$

Then

$$
\left\{d_{N}^{-1} R_{N}(f, t):(f, t) \in \mathcal{F} \times[0,1]\right\}
$$

converges weakly in $\ell^{\infty}(\mathcal{F} \times[0,1])$ in the sense of Definition 3 , to

$$
\begin{aligned}
& \left\{\sum_{j_{1}, \ldots, j_{m}=1}^{p} \tilde{J}_{j_{1}, \ldots, j_{m}}(f) \tilde{K}_{j_{1}, \ldots, j_{m}}(m, D) \int_{\mathbb{R}^{m}}^{\prime \prime} \frac{e^{i t\left(x_{1}+\cdots+x_{m}\right)}-1}{i\left(x_{1}+\cdots+x_{m}\right)} \prod_{j=1}^{m}\left|x_{j}\right|^{-(1-D) / 2}\right. \\
& \left.\times \tilde{B}^{\left(j_{1}\right)}\left(d x_{1}\right) \cdots \tilde{B}^{\left(j_{m}\right)}\left(d x_{m}\right):(f, t) \in \mathcal{F} \times[0,1]\right\} .
\end{aligned}
$$


The measures $\tilde{B}^{(1)}, \ldots, \tilde{B}^{(p)}$ are suitable Hermitian Gaussian random measures, $\tilde{K}_{j_{1}, \ldots, j_{m}}(m$, $D)$ is a normalizing constant, $1 \leq j_{1}, \ldots, j_{m} \leq p$, and

$$
\tilde{J}_{j_{1}, \ldots, j_{m}}(f)=(m !)^{-1} E\left(f\left(\boldsymbol{X}_{1}\right) \prod_{i=1}^{p} H_{i\left(j_{1}, \ldots, j_{m}\right)}\left(X_{1}^{(i)}\right)\right),
$$

where $i\left(j_{1}, \ldots, j_{m}\right)$ is the number of indices $j_{1}, \ldots, j_{m}$ that are equal to $i$.

The limiting process (8) can be interpreted as a generalization of the univariate limit studied in [9], Theorem 1.1, especially the integrand is the same. But due to the fact that each component of $\left(\boldsymbol{X}_{j}\right)_{j \geq 1}$ contributes to the limit, we are faced with $p$ different integrators. More precisely, the above sum includes all possibilities to generate a $m$-fold stochastic integral using $p$ Gaussian random measures. Such integrals were initially studied by Fox and Taqqu [12], Section 2, in the case of real-valued Gaussian random measures. The process (8) appeared first time in a paper by Arcones [2], Theorem 6. He studied Theorem 1 in the non-uniform case, particularly, Arcones proved a functional non-central limit theorem for $\left(f\left(\boldsymbol{X}_{j}\right)\right)_{j \geq 1}, f \in L^{2}\left(\mathcal{N}_{p}\right)$. We have corrected the domain of integration, i.e. we replaced $[-\pi, \pi]^{m}$ by $\mathbb{R}^{m}$, see also [4], Theorem 4.22.

Dehling, Durieu and Tusche [8], Section 3, calculated the bracketing number for a few different classes of functions including indicators of hyperrectangles. In all cases, the bracketing number grows polynomially so that condition (6) is fulfilled. Thus, Theorem 1 implies a multivariate version of Theorem 1.1 by Dehling and Taqqu [9].

Corollary 2. Let $\left(\boldsymbol{X}_{j}\right)_{j \geq 1}$ be a p-dimensional standard Gaussian long-range dependent process in the sense of Definition 2 and let $G: \mathbb{R}^{p} \rightarrow \mathbb{R}^{k}$ be a measurable function. Moreover, let $0<$ $D<1 / m$, where $m$ is the Hermite rank of $\mathcal{F}=\left\{1_{\{G(\cdot) \leq x\}}: x \in \mathbb{R}^{k}\right\}$. Then

$$
\left(d_{N}^{-1} \sum_{j=1}^{\lfloor N t\rfloor}\left(1_{\left\{G\left(\boldsymbol{X}_{j}\right) \leq x\right\}}-P\left(G\left(\boldsymbol{X}_{1}\right) \leq x\right)\right)\right)
$$

converges weakly in $\ell^{\infty}(\mathcal{F} \times[0,1])$ in the sense of Definition 3 , to (8).

We prove Theorem 1 by establishing a weak uniform reduction principle. The formulation is similiar to Theorem 3.1 by Dehling and Taqqu [9]. The notation $P^{*}$ denotes outer probability.

Theorem 3 (Reduction principle). Under the assumptions of Theorem 1, there exist constants $C, \kappa>0$ such that for any $0<\varepsilon \leq 1$

$$
\begin{aligned}
& P^{*}\left(\max _{n \leq N} \sup _{f \in \mathcal{F}} d_{N}^{-1}\left|\sum_{j=1}^{n}\left(f\left(\boldsymbol{X}_{j}\right)-E\left(f\left(\boldsymbol{X}_{1}\right)\right)-\sum_{l_{1}+\cdots+l_{p}=m} \frac{J_{l_{1}, \ldots, l_{p}}(f)}{l_{1} ! \cdots l_{p} !} H_{l_{1}, \ldots, l_{p}}\left(\boldsymbol{X}_{j}\right)\right)\right|>\varepsilon\right) \\
& \quad \leq C N^{-\kappa}\left(1+\varepsilon^{-(2 q+r+1)}\right) .
\end{aligned}
$$


It is well known that $d_{N}$ behaves asymptotically like $N^{1-m D / 2} L^{m / 2}$, see [20], Theorem 3.1. If the cross-covariance is given by (4) instead of (3) one has to normalize with $\operatorname{Var}\left(\sum_{j=1}^{N} H_{m}\left(X_{j}^{(k)}\right)\right)$, where $D_{k}=\min \left\{D_{i}: 1 \leq i \leq p\right\}$. Consequently, only $\sum_{j=1}^{\lfloor N t\rfloor} H_{m}\left(X_{j}^{(k)}\right)$ contributes to the limit because all other terms tend to zero. This behavior was already studied by Leonenko, Sakhno and Taufer [15], Theorem 1b, and reasoned why we focus on a single long-range dependence parameter only. Note, all lemmas in the following section remain valid if we would study (4) instead of (3), only the proof of Lemma 3 has to be slightly modified.

\section{Proofs}

The proof of Theorem 3 is organized as follows. First we prove a moment inequality for partial sums of subordinated Gaussian random vectors (Lemma 2). We can use this result to control the increments of the reduced function-indexed process (Lemma 3). This is essential for proving Lemma 4.

The following lemma by Bardet and Surgailis [3] is the multivariate version of Lemma 4.5 by Taqqu [21]. The term $\varepsilon$-standard used therein denotes a collection of standardized Gaussian random vectors, whose cross-covariance function is bounded by $\varepsilon$. Furthermore, $\sum^{\prime}$ is the sum over all different indices $1 \leq t_{i} \leq n, 1 \leq i \leq p^{\prime}$, such that $t_{i} \neq t_{j}$ for $i \neq j$.

Lemma 1 (Bardet and Surgailis [3], Lemma 1). Let $\left(\boldsymbol{X}_{1}, \ldots, \boldsymbol{X}_{n}\right)$ be a $\varepsilon$-standard Gaussian vector, $\boldsymbol{X}_{t}=\left(X_{t}^{(1)}, \ldots, X_{t}^{(p)}\right) \in \mathbb{R}^{p}, p \geq 1$, and consider a set of functions $f_{j, t, n} \in L^{2}\left(\mathcal{N}_{p}\right)$, $1 \leq j \leq p^{\prime}, p^{\prime} \geq 2,1 \leq t \leq n$. For given integers $m \geq 1,0 \leq \alpha \leq p^{\prime}, n \geq 1$, define

$$
Q_{n}:=\max _{1 \leq t \leq n} \sum_{1 \leq s \leq n, s \neq t} \max _{1 \leq u, v \leq p}\left|E X_{t}^{(u)} X_{s}^{(v)}\right|^{m} .
$$

Assume that the functions $f_{1, t, n}, \ldots, f_{\alpha, t, n}$ have a Hermite rank at least equal to $m$ for any $n \geq 1$, $1 \leq t \leq n$, and that

$$
\varepsilon<\frac{1}{p p^{\prime}-1}
$$

Then

$$
\sum_{t_{1}, \ldots, t_{p^{\prime}}=1}^{n}\left|E\left(f_{1, t_{1}, n}\left(\boldsymbol{X}_{t_{1}}\right) \cdots f_{p^{\prime}, t_{p^{\prime}}, n}\left(\boldsymbol{X}_{t_{p^{\prime}}}\right)\right)\right| \leq C\left(\varepsilon, p^{\prime}, m, \alpha, p\right) K n^{p^{\prime}-\alpha / 2} Q_{n}^{\alpha / 2},
$$

where the constant $C\left(\varepsilon, p^{\prime}, m, \alpha, p\right)$ depends on $\varepsilon, p^{\prime}, m, \alpha, p$ only, and

$$
K=\prod_{j=1}^{p^{\prime}} \max _{1 \leq t \leq n}\left\|f_{j, t, n}\right\|_{2}
$$

For our purposes, it is enough to consider functions $f_{j, t, n}$ which do not depend on $t$ and $n$, that is, $f_{j, t, n}=g_{j}$. In this case the connection to the result of Taqqu [21], Lemma 4.5, becomes 
clearer. An advantage of the above bound is that the constant on the right-hand side is separated into $C\left(\varepsilon, p^{\prime}, m, \alpha, p\right)$ and $K$. This detail will help us to prove the following lemma.

Lemma 2. Let $\left(\boldsymbol{X}_{j}\right)_{j \geq 1}$ be a p-dimensional standard Gaussian process which exhibits longrange dependence in the sense of Definition 2 and let $r^{(i, j)}(k) \leq 1 /(4 p q-1)$ for all $k \in \mathbb{N}$, $1 \leq i, j \leq p$ and some $q \in \mathbb{N}$. Furthermore, let $g: \mathbb{R}^{p} \rightarrow \mathbb{R}$ be a measurable function such that the Hermite rank of $g$ is at least $m$ and $E\left(g\left(X_{1}\right)\right)^{2 q}<\infty$. If $0<D<1 / m$ then for all $n \in \mathbb{N}$ we have

$$
E\left(\sum_{j=1}^{n} g\left(\boldsymbol{X}_{j}\right)\right)^{2 q} \leq C\left(1 \vee\left(E g\left(\boldsymbol{X}_{1}\right)^{2 q}\right)^{q}\right)\left(n \sum_{k=0}^{n} \max _{1 \leq i, j \leq p}\left(r^{(i, j)}(k)\right)^{m}\right)^{q}
$$

Proof. We follow the lines of the proof given in [21], Proposition 4.2.. Using the multinomial theorem, we get

$$
\begin{aligned}
& E\left(\sum_{j=1}^{n} g\left(\boldsymbol{X}_{j}\right)\right)^{2 q} \\
& \quad=\sum_{\substack{k_{1}+\cdots+k_{n}=2 q \\
k_{1}, \ldots, k_{n} \geq 1}} \frac{(2 q) !}{k_{1} ! \cdots k_{n} !} E^{k_{1}}\left(\boldsymbol{X}_{1}\right) \cdots g^{k_{n}}\left(\boldsymbol{X}_{n}\right) \\
& =\sum_{p^{\prime}=1}^{2 q} \sum_{\substack{1 \leq u_{1}<\cdots<u_{p^{\prime}} \leq n k_{u_{1}}+\cdots+k_{u} \\
k_{u_{1}}, \ldots, k_{p_{p^{\prime}}} \geq 1}} \frac{(2 q) !}{k_{u_{1}} ! \cdots k_{u_{p^{\prime}} !} !} \operatorname{Eg}^{k_{u_{1}}\left(\boldsymbol{X}_{u_{1}}\right) \cdots g^{k_{p^{\prime}}}\left(\boldsymbol{X}_{u_{p^{\prime}}}\right)} \\
& \quad \leq \sum_{p^{\prime}=1}^{2 q} \sum_{\substack{v_{1}+\cdots+v_{p^{\prime}}=2 q \\
v_{1}, \ldots, v_{p^{\prime}} \geq 1}}^{n} \sum_{u_{1}, \ldots, u_{p^{\prime}}=1}^{\prime}\left|E g^{v_{1}}\left(\boldsymbol{X}_{u_{1}}\right) \cdots g^{v_{p^{\prime}}}\left(\boldsymbol{X}_{u_{p^{\prime}}}\right)\right| .
\end{aligned}
$$

Let us distinguish two cases, $p^{\prime} \leq q$ and $p^{\prime}>q$, starting with the first one. For $v_{1}, \ldots, v_{p^{\prime}} \geq 1$, satisfying $v_{1}+\cdots+v_{p^{\prime}}=2 q$ we have by Hölder's inequality

$$
\begin{aligned}
\left|E^{v_{1}}\left(\boldsymbol{X}_{u_{1}}\right) \cdots g^{v_{p^{\prime}}}\left(\boldsymbol{X}_{u_{p^{\prime}}}\right)\right| & \leq \prod_{i=1}^{p^{\prime}} \prod_{j=1}^{v_{i}}\left(E g\left(\boldsymbol{X}_{u_{i}}\right)^{2 q}\right)^{1 / 2 q} \\
& =\prod_{i=1}^{p^{\prime}}\left(E g\left(\boldsymbol{X}_{1}\right)^{2 q}\right)^{v_{i} / 2 q} \\
& =E g\left(\boldsymbol{X}_{1}\right)^{2 q}
\end{aligned}
$$


Thus,

$$
\begin{aligned}
& \max _{\substack{v_{1}+\cdots+v_{p^{\prime}}=2 q \\
v_{1}, \ldots, v_{p^{\prime}} \geq 1}} \sum_{u_{1}, \ldots, u_{p^{\prime}}=1}^{n}\left|E^{v_{1}}\left(\boldsymbol{X}_{u_{1}}\right) \cdots g^{v_{p^{\prime}}}\left(\boldsymbol{X}_{u_{p^{\prime}}}\right)\right| \\
& \leq n^{p^{\prime}} \operatorname{Eg}\left(\boldsymbol{X}_{1}\right)^{2 q} \\
& \leq E g\left(\boldsymbol{X}_{1}\right)^{2 q}\left(n \sum_{k=0}^{n} \max _{1 \leq i, j \leq p}\left(r^{(i, j)}(k)\right)^{m}\right)^{q},
\end{aligned}
$$

since $p^{\prime}<q$. To handle the second case, we have to ensure that Lemma 1 can be applied. If $p^{\prime}>q$ and $v_{1}+\cdots+v_{p^{\prime}}=2 q$, then it is impossible that all indices $v_{1}, \ldots, v_{p^{\prime}}$ are greater than one. We separate those which are equal to one as follows

$$
\begin{aligned}
& \max _{\substack{v_{1}+\cdots+v_{p^{\prime}}=2 q \\
v_{1}, \ldots, v_{p^{\prime}} \geq 1}} \sum_{u_{1}, \ldots, u_{p^{\prime}}=1}^{n}\left|E^{v_{1}}\left(\boldsymbol{X}_{u_{1}}\right) \cdots g^{v_{p^{\prime}}}\left(\boldsymbol{X}_{u_{p^{\prime}}}\right)\right| \\
& =\max _{\alpha_{\min } \leq \alpha \leq \alpha_{\max }} \max _{\substack{\alpha+1 \\
v_{\alpha+1}, \cdots+v_{p^{\prime}}=2 q}} \sum_{u_{p^{\prime}} \geq 2}^{n}\left|E g\left(\boldsymbol{X}_{u_{1}}\right) \cdots g\left(\boldsymbol{X}_{u_{\alpha}}\right) g^{v_{\alpha+1}}\left(\boldsymbol{X}_{u_{\alpha+1}}\right) \cdots g^{v_{p^{\prime}}}\left(\boldsymbol{X}_{u_{p^{\prime}}}\right)\right| .
\end{aligned}
$$

The numbers $\alpha_{\min }$ and $\alpha_{\max }$ describe the minimum and maximum number of indices that could be equal to one. More precisely, $\alpha_{\min }=2 p^{\prime}-2 q$ and

$$
\alpha_{\max }= \begin{cases}2 q & \text { if } p^{\prime}=2 q \\ p^{\prime}-1 & \text { otherwise }\end{cases}
$$

For fixed $\alpha$ and $v_{\alpha+1}, \ldots, v_{p^{\prime}}$ we set $g_{i}(\cdot)=g(\cdot)$, if $1 \leq i \leq \alpha$, and $g_{i}(\cdot)=g^{v_{i}}(\cdot)$, if $\alpha+1 \leq i \leq$ $p^{\prime}$. Since $\max \left(v_{\alpha+1}, \ldots, v_{p^{\prime}}\right) \leq q$, we have $g_{i} \in L^{2}\left(\mathcal{N}_{p}\right)$ for all $1 \leq i \leq p^{\prime}$. The Hermite rank of $g_{1}, \ldots, g_{\alpha}$ is at least $m$ and $r^{(i, j)}(k) \leq 1 /(4 p q-1)<1 /\left(p p^{\prime}-1\right)$ for all $k \in \mathbb{N}$. Therefore, the assumptions of Lemma 1 are satisfied with $\varepsilon=1 /(4 p q-1)$ and so

$$
\begin{aligned}
& \sum_{u_{1}, \ldots, u_{p^{\prime}}=1}^{n}\left|E g_{1}\left(\boldsymbol{X}_{u_{1}}\right) \cdots g_{p^{\prime}}\left(\boldsymbol{X}_{u_{p^{\prime}}}\right)\right| \\
& \leq C\left(q, p^{\prime}, m, \alpha, p\right)\left(\prod_{i=1}^{p^{\prime}}\left\|g_{i}\right\|_{2}\right) n^{p^{\prime}-\alpha / 2}\left(\sum_{k=0}^{n} \max _{1 \leq i, j \leq p}\left(r^{(i, j)}(k)\right)^{m}\right)^{\alpha / 2} \\
& \leq C\left(q, p^{\prime}, m, \alpha, p\right)\left(1 \vee\left(E g\left(\boldsymbol{X}_{1}\right)^{2 q}\right)^{q}\right)\left(n \sum_{k=0}^{n} \max _{1 \leq i, j \leq p}\left(r^{(i, j)}(k)\right)^{m}\right)^{q},
\end{aligned}
$$


since $p^{\prime}-\alpha / 2 \leq p^{\prime}-\alpha_{\min } / 2=q$ and $\alpha / 2 \leq \alpha_{\max } / 2 \leq q$. With respect to (9), we get

$$
\begin{aligned}
& E\left(\sum_{j=1}^{n} g\left(\boldsymbol{X}_{j}\right)\right)^{2 q} \\
& \leq C \sum_{p^{\prime}=1}^{2 q} \max _{\substack{v_{1}+\cdots+v_{p^{\prime}}=2 q \\
v_{1}, \ldots, v_{p^{\prime}} \geq 1}} \sum_{u_{1}, \ldots, u_{p^{\prime}}=1}^{n}\left|E g^{v_{1}}\left(\boldsymbol{X}_{u_{1}}\right) \cdots g^{v_{p^{\prime}}}\left(\boldsymbol{X}_{u_{p^{\prime}}}\right)\right| \\
& \leq C\left(n \sum_{k=0}^{n} \max _{1 \leq i, j \leq p}\left(r^{(i, j)}(k)\right)^{m}\right)^{q}\left(q E g\left(\boldsymbol{X}_{1}\right)^{2 q}\right. \\
& \left.\quad+\sum_{p^{\prime}=q+1}^{2 q} \max _{\substack{\alpha_{\min } \leq \alpha \leq \alpha_{\max } \\
v_{\alpha+1}+\cdots+v_{p^{\prime}}=2 q \\
v_{\alpha+1}, \ldots, v_{p^{\prime} \geq 2} \\
v_{1}=\cdots=v_{\alpha}=1}} C\left(q, p^{\prime}, m, \alpha, p\right)\left(1 \vee\left(E g\left(\boldsymbol{X}_{1}\right)^{2 q}\right)^{q}\right)\right) \\
& \leq C\left(1 \vee\left(E g\left(\boldsymbol{X}_{1}\right)^{2 q}\right)^{q}\right)\left(n \sum_{\substack{n \\
1 \leq i, j \leq p}}^{n}\left(r^{(i, j)}(k)\right)^{m}\right)^{q},
\end{aligned}
$$

where $C$ only depends on $p, q$ and $m$.

To simplify the notation, we set

$$
S_{N}(n, f)=d_{N}^{-1} \sum_{j=1}^{n}\left(f\left(\boldsymbol{X}_{j}\right)-E f\left(\boldsymbol{X}_{1}\right)-\sum_{l_{1}+\cdots+l_{p}=m} \frac{J_{l_{1}, \ldots, l_{p}}(f)}{l_{1} ! \cdots l_{p} !} H_{l_{1}, \ldots, l_{p}}\left(\boldsymbol{X}_{j}\right)\right)
$$

and $S_{N}(n, g, h)=S_{N}(n, h)-S_{N}(n, g)$.

Lemma 3. Let the assumptions of Theorem 1 be satisfied and assume further that $r^{(i, j)}(k) \leq$ $1 /(4 p q-1)$ for all $k \in \mathbb{N}, 1 \leq i, j \leq p$. Then there exist positive constants $C$ and $\gamma$ such that for all $g, h \in \mathcal{F}$

$$
\left(N d_{N}^{-1}\right)^{r} E\left(S_{N}(n, g, h)\right)^{2 q} \leq C\left(\frac{n}{N}\right) N^{-\gamma}
$$

Proof. For all $g, h \in \mathcal{F}$ the Hermite rank of

$$
h\left(X_{j}\right)-g\left(X_{j}\right)-E\left(h\left(X_{1}\right)-g\left(X_{1}\right)\right)-\sum_{l_{1}+\cdots+l_{p}=m} \frac{J_{l_{1}, \ldots, l_{p}}(h-g)}{l_{1} ! \cdots l_{p} !} H_{l_{1}, \ldots, l_{p}}\left(X_{j}\right)
$$

is at least $m+1$. By assumption (7) the $2 q$ th moment of these functions are uniformly bounded. Therefore, we can apply Lemma 2. Remember that $d_{N}^{2} \in \mathcal{O}\left(N^{2-m D} L^{m}(N)\right)$, see [20], Theo- 
rem 3.1, and that

$$
n \sum_{k=0}^{n}\left(k^{-D} L(k)\right)^{m+1} \leq C n^{1 \vee(2-(m+1) D)} L^{\prime}(n),
$$

where $L^{\prime}$ is slowly varying at infinity, see [9], page 1777. For simplicity, products of slowly varying functions in $n$ and $N$ will combined into $\boldsymbol{L}(n, N)$. Lemma 2 yields

$$
\begin{aligned}
E\left(S_{N}(n, g, h)\right)^{2 q} & \leq C d_{N}^{-2 q}\left(n \sum_{k=0}^{n} \max _{1 \leq i, j \leq p}\left(r^{(i, j)}(k)\right)^{m+1}\right)^{q} \\
& \leq C d_{N}^{-2 q}\left(n \sum_{k=0}^{n}\left(k^{-D} L(k)\right)^{m+1}\right)^{q} \\
& \leq C d_{N}^{-2 q} n^{q \vee q(2-(m+1) D)} \boldsymbol{L}(n, N) \\
& \leq C N^{q(m D-2)} n^{q \vee q(2-(m+1) D)} \boldsymbol{L}(n, N) \\
& \leq C\left(\frac{n}{N}\right)^{q \vee q(2-(m+1) D)} N^{q(m D-2)} N^{q \vee q(2-(m+1) D)} \boldsymbol{L}(n, N) \\
& \leq C\left(\frac{n}{N}\right) N^{q(m D-1) \vee-q D} \boldsymbol{L}(n, N) .
\end{aligned}
$$

Using the lower bound for $2 q$ from (7), we can conclude the proof, since

$$
\begin{aligned}
& \left(N d_{N}^{-1}\right)^{r} C\left(\frac{n}{N}\right) N^{q(m D-1) \vee-q D} \boldsymbol{L}(n, N) \\
& \leq C N^{m D r / 2}\left(\frac{n}{N}\right) N^{q(m D-1) \vee-q D} \boldsymbol{L}(n, N) \\
& \quad \leq C\left(\frac{n}{N}\right) N^{-\gamma}
\end{aligned}
$$

for an appropriate small $\gamma>0$.

Lemma 4. Let the assumptions of Theorem 1 be satisfied and assume further that $r^{(i, j)}(k) \leq$ $1 /(4 p q-1)$ for all $k \in \mathbb{N}, 1 \leq i, j \leq p$. Then there exist positive constants $\rho$ and $C$ such that for all $n \leq N$ and $0<\varepsilon \leq 1$,

$$
P^{*}\left(\sup _{f \in \mathcal{F}}\left|S_{N}(n, f)\right|>\varepsilon\right) \leq C N^{-\rho}\left(\left(\frac{n}{N}\right) \varepsilon^{-(2 q+r+1)}+\left(\frac{n}{N}\right)^{2-m D}\right) .
$$

Proof. For each $f \in \mathcal{F}$ and each $k \leq K$, where $K$ will be specified in (12), we can find functions $l_{i_{k}(f)}^{(k)}$ and $u_{i_{k}(f)}^{(k)}$ such that $l_{i_{k}(f)}^{(k)} \leq f \leq u_{i_{k}(f)}^{(k)}$ and $\left\|u_{i_{k}(f)}^{(k)}-l_{i_{k}(f)}^{(k)}\right\|_{2} \leq 2^{-k}$. Using the lower 
functions, we get the telescoping sum

$$
\begin{aligned}
S_{N}(n, f)= & S_{N}\left(n, l_{i_{0}(f)}^{(0)}\right)+S_{N}\left(n, l_{i_{0}(f)}^{(0)}, l_{i_{1}(f)}^{(1)}\right)+S_{N}\left(n, l_{i_{1}(f)}^{(1)}, l_{i_{2}(f)}^{(2)}\right)+\cdots \\
& +S_{N}\left(n, l_{i_{K-1}(f)}^{(K-1)}, l_{i_{K}(f)}^{(K)}\right)+S_{N}\left(n, l_{i_{K}(f)}^{(K)}, f\right) .
\end{aligned}
$$

The last term can be handled as follows

$$
\begin{aligned}
& \left|S_{N}\left(n, l_{i_{K}(f)}^{(K)}, f\right)\right| \\
& =\mid d_{N}^{-1} \sum_{j=1}^{n}\left(f\left(\boldsymbol{X}_{j}\right)-l_{i_{K}(f)}^{(K)}\left(\boldsymbol{X}_{j}\right)-E\left(f\left(\boldsymbol{X}_{1}\right)-l_{i_{K}(f)}^{(K)}\left(\boldsymbol{X}_{1}\right)\right)\right. \\
& \left.-\sum_{l_{1}+\cdots+l_{p}=m} \frac{J_{l_{1}, \ldots, l_{p}}(f)-J_{l_{1}, \ldots, l_{p}}\left(l_{i_{K}(f)}^{(K)}\right)}{l_{1} ! \cdots l_{p} !} H_{l_{1}, \ldots, l_{p}}\left(\boldsymbol{X}_{j}\right)\right) \\
& \leq d_{N}^{-1} \sum_{j=1}^{n}\left(f\left(\boldsymbol{X}_{j}\right)-l_{i_{K}(f)}^{(K)}\left(\boldsymbol{X}_{j}\right)+E\left(f\left(\boldsymbol{X}_{1}\right)-l_{i_{K}(f)}^{(K)}\left(\boldsymbol{X}_{1}\right)\right)\right) \\
& +\sum_{l_{1}+\cdots+l_{p}=m}\left|\frac{E\left(\left(f\left(\boldsymbol{X}_{1}\right)-l_{i_{K}(f)}^{(K)}\left(\boldsymbol{X}_{1}\right)\right) H_{l_{1}, \ldots, l_{p}}\left(\boldsymbol{X}_{1}\right)\right)}{l_{1} ! \cdots l_{p} !}\right| d_{N}^{-1}\left|\sum_{j=1}^{n} H_{l_{1}, \ldots, l_{p}}\left(\boldsymbol{X}_{j}\right)\right| \\
& \leq d_{N}^{-1} \sum_{j=1}^{n}\left(u_{i_{K}(f)}^{(K)}\left(\boldsymbol{X}_{j}\right)-l_{i_{K}(f)}^{(K)}\left(\boldsymbol{X}_{j}\right)+E\left(u_{i_{K}(f)}^{(K)}\left(\boldsymbol{X}_{1}\right)-l_{i_{K}(f)}^{(K)}\left(\boldsymbol{X}_{1}\right)\right)\right) \\
& +\sum_{l_{1}+\cdots+l_{p}=m}\left|\frac{E\left(\left|u_{i_{K}(f)}^{(K)}\left(\boldsymbol{X}_{1}\right)-l_{i_{K}(f)}^{(K)}\left(\boldsymbol{X}_{1}\right)\right| \cdot\left|H_{l_{1}, \ldots, l_{p}}\left(\boldsymbol{X}_{1}\right)\right|\right)}{l_{1} ! \cdots l_{p} !}\right| d_{N}^{-1}\left|\sum_{j=1}^{n} H_{l_{1}, \ldots, l_{p}}\left(\boldsymbol{X}_{j}\right)\right| \\
& \leq S_{N}\left(n, l_{i_{K}(f)}^{(K)}, u_{i_{K}(f)}^{(K)}\right)+2 n d_{N}^{-1} E\left(u_{i_{K}(f)}^{(K)}\left(\boldsymbol{X}_{1}\right)-l_{i_{K}(f)}^{(K)}\left(\boldsymbol{X}_{1}\right)\right) \\
& +2 \cdot \sum_{l_{1}+\cdots+l_{p}=m}\left|\frac{E\left(\left|u_{i_{K}(f)}^{(K)}\left(\boldsymbol{X}_{1}\right)-l_{i_{K}(f)}^{(K)}\left(\boldsymbol{X}_{1}\right)\right| \cdot\left|H_{l_{1}, \ldots, l_{p}}\left(\boldsymbol{X}_{1}\right)\right|\right)}{l_{1} ! \cdots l_{p} !}\right| \\
& \cdot d_{N}^{-1}\left|\sum_{j=1}^{n} H_{l_{1}, \ldots, l_{p}}\left(\boldsymbol{X}_{j}\right)\right| \\
& \leq S_{N}\left(n, l_{i_{K}(f)}^{(K)}, u_{i_{K}(f)}^{(K)}\right)+2 n d_{N}^{-1} 2^{-K}+2 \cdot 2^{-K} d_{N}^{-1} \cdot \sum_{l_{1}+\cdots+l_{p}=m}\left|\sum_{j=1}^{n} H_{l_{1}, \ldots, l_{p}}\left(\boldsymbol{X}_{j}\right)\right| \text {, }
\end{aligned}
$$


by using Cauchy-Schwarz inequality. Since $\varepsilon / 2+\sum_{k=0}^{K} \varepsilon /(k+3)^{2}<\varepsilon$ for all $K \in \mathbb{N}$, we have

$$
\begin{aligned}
& P^{*}\left(\sup _{f \in \mathcal{F}}\left|S_{N}(n, f)\right|>\varepsilon\right) \\
& \leq P^{*}\left(\max _{f \in \mathcal{F}}\left|S_{N}\left(n, l_{i_{0}(f)}^{(0)}\right)\right|>\varepsilon / 9\right) \\
&+P^{*}\left(\max _{f \in \mathcal{F}}\left|S_{N}\left(n, l_{i_{0}(f)}^{(0)}, l_{i_{1}(f)}^{(1)}\right)\right|>\varepsilon / 16\right)+\cdots \\
&+P^{*}\left(\max _{f \in \mathcal{F}}\left|S_{N}\left(n, l_{i_{K-1}(f)}^{(K-1)}, l_{i_{K}(f)}^{(K)}\right)\right|>\varepsilon /(K+3)^{2}\right) \\
&+P^{*}\left(\max _{f \in \mathcal{F}}\left|S_{N}\left(n, l_{i_{K}(f)}^{(K)}, u_{i_{K}(f)}^{(K)}\right)\right|>\varepsilon /(K+4)^{2}\right) \\
&+P\left(2 \cdot 2^{-K} d_{N}^{-1} \sum_{l_{1}+\cdots+l_{p}=m}\left|\sum_{j=1}^{n} H_{l_{1}, \ldots, l_{p}}\left(\boldsymbol{X}_{j}\right)\right|>\varepsilon / 2-2 n d_{N}^{-1} 2^{-K}\right) .
\end{aligned}
$$

Set

$$
\begin{aligned}
& M=\left|\left\{\left(l_{1}, \ldots, l_{p}\right) \in \mathbb{N}^{p}: l_{1}+\cdots l_{p}=m\right\}\right|=\left(\begin{array}{c}
m+p-1 \\
m
\end{array}\right), \\
& K=\left\lceil\log _{2}\left(\frac{8 N d_{N}^{-1}}{\varepsilon}\right)\right]
\end{aligned}
$$

and let $N_{k}$ be the bracketing number with respect to $2^{-k}$. Applying Lemma 3 yields

$$
\begin{aligned}
& P^{*}\left(\max _{f \in \mathcal{F}}\left|S_{N}\left(n, l_{i_{k-1}(f)}^{(k-1)}, l_{i_{k}(f)}^{(k)}\right)\right|>\varepsilon /(k+3)^{2}\right) \\
& \leq \sum_{s=1}^{N_{k-1}} \sum_{u=1}^{N_{k}} P\left(\left|S_{N}\left(n, l_{s}^{(k-1)}, l_{u}^{(k)}\right)\right|>\varepsilon /(k+3)^{2}\right) \\
& \leq \sum_{s=1}^{N_{k-1}} \sum_{u=1}^{N_{k}}(k+3)^{4 q} \varepsilon^{-2 q} E\left|S_{N}\left(n, l_{s}^{(k-1)}, l_{u}^{(k)}\right)\right|^{2 q} \\
& \leq C(k+3)^{4 q} N_{k}^{2} \varepsilon^{-2 q}\left(N d_{N}^{-1}\right)^{-r}\left(\frac{n}{N}\right) N^{-\gamma}
\end{aligned}
$$

for $1 \leq k \leq K$. In the same way, we get

$$
\begin{aligned}
& P^{*}\left(\max _{f \in \mathcal{F}}\left|S_{N}\left(n, l_{i_{0}(f)}^{(0)}\right)\right|>\varepsilon / 9\right) \\
& \quad \leq C 3^{4 q} N_{1} \varepsilon^{-2 q}\left(N d_{N}^{-1}\right)^{-r}\left(\frac{n}{N}\right) N^{-\gamma}
\end{aligned}
$$


and

$$
\begin{aligned}
& P^{*}\left(\max _{f \in \mathcal{F}}\left|S_{N}\left(n, l_{i_{K}(f)}^{(K)}, u_{i_{K}(f)}^{(K)}\right)\right|>\varepsilon /(K+4)^{2}\right) \\
& \quad \leq C(K+4)^{4 q} N_{K} \varepsilon^{-2 q}\left(N d_{N}^{-1}\right)^{-r}\left(\frac{n}{N}\right) N^{-\gamma}
\end{aligned}
$$

Let $M$ be the quantity given by (11). Since (12) implies $(\varepsilon / 4)^{-2}<2^{2 K-2} N^{-2} d_{N}^{2}$ we have

$$
\begin{aligned}
& P\left(2 \cdot 2^{-K} d_{N}^{-1} \cdot \sum_{l_{1}+\cdots+l_{p}=m}\left|\sum_{j=1}^{n} H_{l_{1}, \ldots, l_{p}}\left(\boldsymbol{X}_{j}\right)\right|>\varepsilon / 2-2 n d_{N}^{-1} 2^{-K}\right) \\
& \quad \leq P\left(d_{N}^{-1} \cdot \sum_{l_{1}+\cdots+l_{p}=m}\left|\sum_{j=1}^{n} H_{l_{1}, \ldots, l_{p}}\left(\boldsymbol{X}_{j}\right)\right|>\frac{2^{K-1} \varepsilon}{4}\right) \\
& \leq \sum_{l_{1}+\cdots+l_{p}=m} P\left(d_{N}^{-1}\left|\sum_{j=1}^{n} H_{l_{1}, \ldots, l_{p}}\left(\boldsymbol{X}_{j}\right)\right|>\frac{2^{K-1} \varepsilon}{4 M}\right) \\
& \quad \leq d_{N}^{-2} \sum_{l_{1}+\cdots+l_{p}=m} E\left|\sum_{j=1}^{n} H_{l_{1}, \ldots, l_{p}}\left(\boldsymbol{X}_{j}\right)\right|^{2}\left(\frac{\varepsilon}{4}\right)^{-2} 2^{-2 K+2} M^{2} \\
& \leq C d_{N}^{-2} n^{2-m D} L(b n)^{m} N^{-2} d_{N}^{2} \\
& \leq C\left(\frac{n}{N}\right)^{2-m D}\left(\frac{L(b n)}{L(N)}\right)^{m} N^{-m D} L^{m}(N) \\
& \quad \leq C\left(\frac{n}{N}\right)^{2-m D} N^{-m D+\lambda}
\end{aligned}
$$

for any $\lambda>0$. Thus, assumption (6) and (12) can be used to bound (10) finally in the following way

$$
\begin{aligned}
& P^{*}\left(\sup _{f \in \mathcal{F}}\left|S_{N}(n, f)\right|>\varepsilon\right) \\
& \quad \leq C \varepsilon^{-2 q}\left(N d_{N}^{-1}\right)^{-r}\left(\frac{n}{N}\right) N^{-\gamma} \sum_{k=0}^{K}(k+3)^{4 q} N_{k}^{2}+C\left(\frac{n}{N}\right)^{2-m D} N^{-m D+\lambda} \\
& \quad=C \varepsilon^{-2 q}\left(N d_{N}^{-1}\right)^{-r}\left(\frac{n}{N}\right) N^{-\gamma} \sum_{k=0}^{K} 2^{-r k} 2^{r k}(k+3)^{4 q} N_{k}^{2}+C\left(\frac{n}{N}\right)^{2-m D} N^{-m D+\lambda}
\end{aligned}
$$




$$
\begin{aligned}
& \leq C \varepsilon^{-2 q}\left(N d_{N}^{-1}\right)^{-r}\left(\frac{n}{N}\right) N^{-\gamma}\left(2^{K}\right)^{r}(K+3)^{4 q} \sum_{k=0}^{\infty} 2^{-r k} N_{k}^{2}+C\left(\frac{n}{N}\right)^{2-m D} N^{-m D+\lambda} \\
& \leq C \varepsilon^{-2 q}\left(N d_{N}^{-1}\right)^{-r}\left(\frac{n}{N}\right) N^{-\gamma} \varepsilon^{-r}\left(N d_{N}^{-1}\right)^{r}(K+3)^{4 q}+C\left(\frac{n}{N}\right)^{2-m D} N^{-m D+\lambda} \\
& \leq C\left(\frac{n}{N}\right) N^{-\gamma}(K+3)^{4 q} \varepsilon^{-(2 q+r)}+C\left(\frac{n}{N}\right)^{2-m D} N^{-m D+\lambda} \\
& \leq C N^{-\rho}\left(\left(\frac{n}{N}\right) \varepsilon^{-(2 q+r+1)}+\left(\frac{n}{N}\right)^{2-m D}\right)
\end{aligned}
$$

for a sufficient small $\rho$. Note, for the last inequality, we use $(K+3)^{4 q} \leq C \varepsilon^{-1} N^{\delta}$ for any $\delta>0$.

Proof of Theorem 3. If the cross-covariance function satisfies $r^{(i, j)}(k) \leq 1 /(4 p q-1)$ for all $k \in \mathbb{N}$ and $1 \leq i, j \leq p$ then Lemma 4 holds and Theorem 3 follows by adapting the proof given in [9], page 1781. The general case can be treated as in [21], page 225. Choose $b \in \mathbb{N}$ such that $r^{(i, j)}(k) \leq 1 /(4 p q-1)$ for all $k \geq b$ and set

$$
\tilde{f}\left(\boldsymbol{X}_{j}\right)=f\left(\boldsymbol{X}_{j}\right)-E f\left(\boldsymbol{X}_{1}\right)-\sum_{l_{1}+\cdots+l_{p}=m} \frac{J_{l_{1}, \ldots, l_{p}}(f)}{l_{1} ! \cdots l_{p} !} H_{l_{1}, \ldots, l_{p}}\left(\boldsymbol{X}_{j}\right)
$$

We can decompose $S_{N}(n, f)$ as follows

$$
\begin{aligned}
\left|S_{N}(n, f)\right| & \leq d_{N}^{-1}\left(\sum_{j=1}^{j^{*}}\left|\sum_{k=0}^{\left\lfloor\frac{n}{b}\right\rfloor} \tilde{f}\left(\boldsymbol{X}_{j+k b}\right)\right|+\sum_{j=j^{*}+1}^{b}\left|\sum_{k=0}^{\left\lfloor\frac{n}{b}\right\rfloor-1} \tilde{f}\left(\boldsymbol{X}_{j+k b}\right)\right|\right) \\
& \leq \sum_{j=1}^{b} \max _{1 \leq l \leq N / b} d_{N}^{-1}\left|\sum_{k=0}^{l} \tilde{f}\left(\boldsymbol{X}_{j+k b}\right)\right|
\end{aligned}
$$

where $j^{*}=b$, if $n / b$ is an integer, and $j^{*}=n-\lfloor n / b\rfloor b$ otherwise. Taking the supremum of both sides yields

$$
\max _{1 \leq n \leq N} \sup _{f \in \mathcal{F}}\left|S_{N}(n, f)\right| \leq \sum_{j=1}^{b} \max _{1 \leq l \leq N} \sup _{f \in \mathcal{F}} d_{N}^{-1}\left|\sum_{k=1}^{l} \tilde{f}\left(\boldsymbol{X}_{j+(k-1) b}\right)\right|
$$


For any $j$ the series $\left(\boldsymbol{X}_{j+(k-1) b}\right)_{k \geq 1}$ satisfies the assumption of Lemma 4. Therefore,

$$
\begin{aligned}
& P^{*}\left(\max _{1 \leq n \leq N} \sup _{f \in \mathcal{F}}\left|S_{N}(n, f)\right|>\varepsilon\right) \\
& \quad \leq \sum_{j=1}^{b} P^{*}\left(\max _{1 \leq l \leq N} \sup _{f \in \mathcal{F}} d_{N}^{-1}\left|\sum_{k=1}^{l} \tilde{f}\left(\boldsymbol{X}_{j+(k-1) b}\right)\right|>\varepsilon / b\right) \\
& \quad \leq C N^{-\kappa}\left(1+\varepsilon^{-(2 q+r+1)}\right) .
\end{aligned}
$$

Lemma 5. For all $m \in \mathbb{N}$ and $a_{1}, \ldots, a_{p} \in \mathbb{R}$ with $a_{1}^{2}+\cdots+a_{p}^{2}=1$ we have

$$
H_{m}\left(\sum_{j=1}^{p} a_{j} x_{j}\right)=\sum_{m_{1}+\cdots+m_{p}=m} \frac{m !}{m_{1} ! \cdots m_{p} !} \prod_{j=1}^{p} a_{j}^{m_{j}} H_{m_{j}}\left(x_{j}\right) .
$$

Since we could not find a proof for this well known result in the literature, see, for example, [2], page 2255, and [4], page 113, we give one here.

Proof. We first show that all partial derivatives are equal by using induction. For $m=1$, this is obvious. Since $H_{n}^{\prime}(x)=n H_{n-1}(x)$ we have

$$
\begin{aligned}
& \frac{\partial}{\partial x_{1}}\left(\sum_{m_{1}+\cdots+m_{p}=m+1} \frac{(m+1) !}{m_{1} ! \cdots m_{p} !} \prod_{j=1}^{p} a_{j}^{m_{j}} H_{m_{j}}\left(x_{j}\right)\right) \\
& =\sum_{m_{1}+\cdots+m_{p}=m+1} \frac{(m+1) !}{\left(m_{1}-1\right) ! \cdots m_{p} !} a_{1}^{m_{1}} H_{m_{1}-1}\left(x_{1}\right) \prod_{j=2}^{p} a_{j}^{m_{j}} H_{m_{j}}\left(x_{j}\right) \\
& =a_{1}(m+1) \sum_{m_{1}+\cdots+m_{p}=m} \frac{(m) !}{m_{1} ! \cdots m_{p} !} \prod_{j=1}^{p} a_{j}^{m_{j}} H_{m_{j}}\left(x_{j}\right) \\
& =a_{1}(m+1) H_{m}\left(\sum_{j=1}^{p} a_{j} x_{j}\right) \\
& =\frac{\partial}{\partial x_{1}} H_{m+1}\left(\sum_{j=1}^{p} a_{j} x_{j}\right) .
\end{aligned}
$$

The other derivatives can be handled similarly. Therefore, (13) holds up to a constant. Let $x_{1}=$ $\cdots=x_{p}=0$. If $m$ is odd, both sides of (13) are equal to zero and thus the constant vanishes. For even $m$ we have $H_{m}(0)=(-1)^{m / 2}(m-1) !$ !, where

$$
(m-1) ! !:=(m-1)(m-3) \cdots 3 \cdot 1=\frac{m !}{2^{m / 2}(m / 2) !} .
$$


This yields

$$
\begin{aligned}
& \sum_{m_{1}+\cdots+m_{p}=m} \frac{m !}{m_{1} ! \cdots m_{p} !} \prod_{j=1}^{p} a_{j}^{m_{j}} H_{m_{j}}(0) \\
& =\sum_{2 m_{1}+\cdots+2 m_{p}=m} \frac{m !}{\left(2 m_{1}\right) ! \cdots\left(2 m_{p}\right) !} \prod_{j=1}^{p}(-1)^{m_{j}} a_{j}^{2 m_{j}}\left(2 m_{j}-1\right) ! ! \\
& =(-1)^{m / 2} \sum_{2 m_{1}+\cdots+2 m_{p}=m} \frac{m !}{\left(2 m_{1}\right) ! ! \cdots\left(2 m_{p}\right) ! !} \prod_{j=1}^{p}\left(a_{j}^{2}\right)^{m_{j}} \\
& =(-1)^{m / 2} \sum_{m_{1}+\cdots+m_{p}=m / 2} \frac{m !}{2^{m / 2} m_{1} ! \cdots m_{p} !} \prod_{j=1}^{p}\left(a_{j}^{2}\right)^{m_{j}} \\
& =(-1)^{m / 2} \sum_{m_{1}+\cdots+m_{p}=m / 2} \frac{(m-1) ! ! 2^{m / 2}(m / 2) !}{2^{m / 2} m_{1} ! \cdots m_{p} !} \prod_{j=1}^{p}\left(a_{j}^{2}\right)^{m_{j}} \\
& =(-1)^{m / 2}(m-1) ! ! \sum_{m_{1}+\cdots+m_{p}=m / 2} \frac{(m / 2) !}{m_{1} ! \cdots m_{p} !} \prod_{j=1}^{p}\left(a_{j}^{2}\right)^{m_{j}} \\
& =H_{m}(0)\left(\sum_{j=1}^{p} a_{j}^{2}\right)^{m / 2} \\
& =H_{m}(0) \text {. }
\end{aligned}
$$

Proof of Theorem 1. By Theorem 3, it is enough to study the limit of

$$
\left\{d_{N}^{-1} \sum_{j=1}^{\lfloor N t\rfloor} \sum_{l_{1}+\cdots+l_{p}=m} \frac{J_{l_{1}, \ldots, l_{p}}(f)}{l_{1} ! \cdots l_{p} !} H_{l_{1}, \ldots, l_{p}}\left(\boldsymbol{X}_{j}\right):(f, t) \in \mathcal{F} \times[0,1]\right\} .
$$

We first show that in the current situation Lemma 5 can be applied. By independence of multivariate monomials of degree $m$, we can find for all $k_{1}, \ldots, k_{p}$ satisfying $k_{1}+\cdots+k_{p}=m$, real numbers $a_{k_{1}, \ldots, k_{p}}^{(1)}, \ldots a_{k_{1}, \ldots, k_{p}}^{(p)}$, such that the $\left(\begin{array}{c}m+p-1 \\ m\end{array}\right) \times\left(\begin{array}{c}m+p-1 \\ m\end{array}\right)$ matrix

$$
A=\left(\prod_{i=1}^{p}\left(a_{k_{1}, \ldots, k_{p}}^{(i)}\right)^{m_{i}}\right)_{\substack{m_{1}+\cdots+m_{p}=m \\ k_{1}+\cdots+k_{p}=m}}
$$

is invertible. More precisely, sorting the tuples $\left(m_{1}, \ldots, m_{p}\right), m_{1}+\cdots+m_{p}=m$ and $\left(k_{1}, \ldots, k_{p}\right), k_{1}+\cdots+k_{p}=m$ lexicographically, the entry $a_{q_{1}, q_{2}}$ of $A$ is given by 
$\prod_{i=1}^{p}\left(a_{k_{1}, \ldots, k_{p}}^{(i)}\right)^{m_{i}}$ with respect to this order. After normalization, we have $\sum_{i=1}^{p}\left(a_{k_{1}, \ldots, k_{p}}^{(i)}\right)^{2}=1$.

For a suitable diagonal matrix $M$ of the same size define $B:=M A^{-1}$ such that

$$
\begin{aligned}
& \sum_{k_{1}+\cdots+k_{p}=m} b\left(k_{1}, \ldots, k_{p}, l_{1}, \ldots, l_{p}\right)\left(a_{k_{1}, \ldots, k_{p}}^{(1)}\right)^{m_{1}} \cdots\left(a_{k_{1}, \ldots, k_{p}}^{(p)}\right)^{m_{p}} \\
& = \begin{cases}(m !)^{-1} \prod_{i=1}^{p} l_{i} ! & \text { if }\left(m_{1}, \ldots, m_{p}\right)=\left(l_{1}, \ldots, l_{p}\right), \\
0 & \text { otherwise, }\end{cases}
\end{aligned}
$$

where $b\left(k_{1}, \ldots, k_{p}, l_{1}, \ldots, l_{p}\right)$ denotes that entry of $B$ whose row and column number is given by the lexicographical order of $k_{1}, \ldots, k_{p}$ and $l_{1}, \ldots, l_{p}$. Using Lemma 5 together with (14) we get

$$
\begin{aligned}
& \sum_{\substack{l_{1}+\cdots+l_{p}=m \\
k_{1}+\cdots+k_{p}=m}} J_{l_{1}, \ldots, l_{p}}(f)\left(\prod_{i=1}^{p}\left(l_{i} !\right)^{-1}\right) b\left(k_{1}, \ldots, k_{p}, l_{1}, \ldots, l_{p}\right) H_{m}\left(\sum_{i=1}^{p} a_{k_{1}, \ldots, k_{p}}^{(i)} X_{j}^{(i)}\right) \\
& =\sum_{\substack{l_{1}+\cdots+l_{p}=m \\
k_{1}+\cdots+k_{p}=m}} \sum_{m_{1}+\cdots+m_{p}=m} J_{l_{1}, \ldots, l_{p}}(f)\left(\prod_{i=1}^{p}\left(l_{i} !\right)^{-1}\right) b\left(k_{1}, \ldots, k_{p}, l_{1}, \ldots, l_{p}\right) \\
& \quad \times m ! \prod_{i=1}^{p}\left(m_{i} !\right)^{-1}\left(a_{k_{1}, \ldots, k_{p}}^{(i)}\right)^{m_{i}} H_{m_{i}}\left(X_{j}^{(i)}\right) \\
& \sum_{l_{1}+\cdots+l_{p}=m} J_{l_{1}, \ldots, l_{p}}(f) \prod_{i=1}^{p}\left(l_{i} !\right)^{-1} H_{l_{i}}\left(X_{j}^{(i)}\right) .
\end{aligned}
$$

Define for simplicity

$$
I\left(f ; k_{1}, \ldots, k_{p}\right):=\sum_{l_{1}+\cdots+l_{p}=m} J_{l_{1}, \ldots, l_{p}}(f)\left(\prod_{i=1}^{p}\left(l_{i} !\right)^{-1}\right) b\left(k_{1}, \ldots, k_{p}, l_{1}, \ldots, l_{p}\right),
$$

whereby the following identity holds

$$
\begin{aligned}
& d_{N}^{-1} \sum_{j=1}^{\lfloor N t\rfloor} \sum_{l_{1}+\cdots+l_{p}=m} J_{l_{1}, \ldots, l_{p}}(f) \prod_{i=1}^{p}\left(l_{i} !\right)^{-1} H_{l_{i}}\left(X_{j}^{(i)}\right) \\
& \quad=d_{N}^{-1} \sum_{j=1}^{\lfloor N t\rfloor} \sum_{k_{1}+\cdots+k_{p}=m} I\left(f ; k_{1}, \ldots, k_{p}\right) H_{m}\left(\sum_{i=1}^{p} a_{k_{1}, \ldots, k_{p}}^{(i)} X_{j}^{(i)}\right) .
\end{aligned}
$$


As stated by Arcones [2], (3.6), there exist Hermitian Gaussian random measures $\tilde{B}^{(1)}, \ldots, \tilde{B}^{(p)}$, such that

$$
\begin{aligned}
& \left\{d_{N}^{-1} \sum_{j=1}^{\lfloor N t\rfloor}\left(H_{m}\left(X_{j}^{(1)}\right), \ldots, H_{m}\left(X_{j}^{(p)}\right)\right): 0 \leq t \leq 1\right\} \\
& \stackrel{d}{\longrightarrow}\left\{\left(Z_{m}^{(1)}(t), \ldots,\left(Z_{m}^{(p)}(t)\right): 0 \leq t \leq 1\right\},\right.
\end{aligned}
$$

in $(D[0,1])^{p}$, where the processes $\left(Z_{m}^{(j)}(t)\right)$ are up to a constant the Hermite processes defined by (2) with respect to $\tilde{B}^{(j)}$. More precisely, if $\boldsymbol{Z}=\left(Z^{(1)}, \ldots, Z^{(p)}\right)$ is the vector-valued random spectral measure such that

$$
\boldsymbol{X}_{k}=\int_{-\pi}^{\pi} e^{i k x} \boldsymbol{Z}(d x),
$$

see, for example, [6], Theorem 11.8.2, then for any bounded symmetric intervals $A_{1}, \ldots, A_{p} \subset \mathbb{R}$, the random vector $\left(\tilde{B}^{(1)}\left(A_{1}\right), \ldots, \tilde{B}^{(p)}\left(A_{p}\right)\right)$ is the weak limit of $L^{-1 / 2}(N) N^{D / 2}\left(Z^{(1)}\left(N^{-1} A_{1}\right), \ldots, Z^{(p)}\left(N^{-1} A_{p}\right)\right)$, see [13], page 1172 .

Note that for any integrable function $h$

$$
\begin{aligned}
& \int_{\mathbb{R}^{m}}^{\prime \prime} h\left(x_{1}, \ldots, x_{m}\right)\left(\sum_{i=1}^{p} a_{k_{1}, \ldots, k_{p}}^{(i)} \tilde{B}^{(i)}\right)\left(d x_{1}\right) \cdots\left(\sum_{i=1}^{p} a_{k_{1}, \ldots, k_{p}}^{(i)} \tilde{B}^{(i)}\right)\left(d x_{m}\right) \\
& =\sum_{j_{1}, \ldots, j_{m}=1}^{p} a_{k_{1}, \ldots, k_{p}}^{\left(j_{1}\right)} \cdots a_{k_{1}, \ldots, k_{p}}^{\left(j_{m}\right)} \int_{\mathbb{R}^{m}}^{\prime \prime} h\left(x_{1}, \ldots, x_{m}\right) \tilde{B}^{\left(j_{1}\right)}\left(d x_{1}\right) \cdots \tilde{B}^{\left(j_{m}\right)}\left(d x_{m}\right) .
\end{aligned}
$$

and therefore

$$
\begin{aligned}
& \left\{d_{N}^{-1} \sum_{j=1}^{\lfloor N t\rfloor} H_{m}\left(\sum_{i=1}^{p} a_{k_{1}, \ldots, k_{p}}^{(i)} X_{j}^{(i)}\right): k_{1}, \ldots, k_{p}=m, t \in[0,1]\right\} \\
& \stackrel{d}{\longrightarrow}\left\{\sum_{j_{1}, \ldots, j_{m}=1}^{p} a_{k_{1}, \ldots, k_{p}}^{\left(j_{1}\right)} \cdots a_{k_{1}, \ldots, k_{p}}^{\left(j_{m}\right)} Z_{j_{1}, \ldots, j_{m}}(t): k_{1}, \ldots, k_{p}=m, t \in[0,1]\right\},
\end{aligned}
$$

where

$$
\begin{aligned}
& Z_{j_{1}, \ldots, j_{m}}(t) \\
& \quad=\tilde{K}_{j_{1}, \ldots, j_{m}}(m, D) \int_{\mathbb{R}^{m}}^{\prime \prime} \frac{e^{i t\left(x_{1}+\cdots+x_{m}\right)}-1}{i\left(x_{1}+\cdots+x_{m}\right)} \prod_{j=1}^{m}\left|x_{j}\right|^{-(1-D) / 2} \tilde{B}^{\left(j_{1}\right)}\left(d x_{1}\right) \cdots \tilde{B}^{\left(j_{m}\right)}\left(d x_{m}\right)
\end{aligned}
$$

and $\tilde{K}_{j_{1}, \ldots, j_{m}}(m, D)$ is a suitable constant. Using the almost sure representation theorem, see [19], page 71 , we can find suitable versions $\left(\tilde{S}_{N}(t)\right)_{N \geq 1}$ and $(\tilde{Z}(t))$ such that this convergence 
holds almost surely. Since the functions $I\left(f ; k_{1}, \ldots, k_{p}\right), k_{1}+\cdots+k_{p}=m$, are bounded we have

$$
\begin{aligned}
& \left\{d_{N}^{-1} I\left(f ; k_{1}, \ldots, k_{p}\right) \tilde{S}_{N}^{\left(k_{1}, \ldots, k_{p}\right)}(t): k_{1}+\cdots+k_{p}=m, f \in \mathcal{F}, t \in[0,1]\right\} \\
& \quad \stackrel{\text { a.s. }}{\longrightarrow}\left\{I\left(f ; k_{1}, \ldots, k_{p}\right) \tilde{Z}^{\left(k_{1}, \ldots, k_{p}\right)}(t): k_{1}+\cdots+k_{p}=m, f \in \mathcal{F}, t \in[0,1]\right\}
\end{aligned}
$$

and consequently

$$
\begin{aligned}
& \left\{d_{N}^{-1} \sum_{j=1}^{\lfloor N t\rfloor} \sum_{k_{1}+\cdots+k_{p}=m} I\left(f ; k_{1}, \ldots, k_{p}\right) H_{m}\left(\sum_{i=1}^{p} a_{k_{1}, \ldots, k_{p}}^{(i)} X_{j}^{(i)}\right): f \in \mathcal{F}, t \in[0,1]\right\} \\
& \stackrel{d}{\longrightarrow}\left\{\sum_{j_{1}, \ldots, j_{m}=1}^{p} \sum_{k_{1}+\cdots+k_{p}=m} a_{k_{1}, \ldots, k_{p}}^{\left(j_{1}\right)} \cdots a_{k_{1}, \ldots, k_{p}}^{\left(j_{m}\right)} I\left(f ; k_{1}, \ldots, k_{p}\right) Z_{j_{1}, \ldots, j_{m}}(t):\right. \\
& \quad f \in \mathcal{F}, t \in[0,1]\}
\end{aligned}
$$

in $\ell^{\infty}(\mathcal{F} \times[0,1])$. To conclude, we have to verify that the limiting process is the one stated in Theorem 1.

$$
\begin{aligned}
& \sum_{j_{1}, \ldots, j_{m}=1}^{p} \sum_{k_{1}+\cdots+k_{p}=m} I\left(f ; k_{1}, \ldots, k_{p}\right) a_{k_{1}, \ldots, k_{p}}^{\left(j_{1}\right)} \cdots a_{k_{1}, \ldots, k_{p}}^{\left(j_{m}\right)} \\
& =\sum_{j_{1}, \ldots, j_{m}=1}^{p} \sum_{\substack{k_{1}+\cdots+k_{p}=m \\
l_{1}+\cdots+l_{p}=m}} J_{l_{1}, \ldots, l_{p}}(f)\left(\prod_{i=1}^{p}\left(l_{i} !\right)^{-1}\right) b\left(k_{1}, \ldots, k_{p}, l_{1}, \ldots, l_{p}\right) a_{k_{1}, \ldots, k_{p}}^{\left(j_{1}\right)} \cdots a_{k_{1}, \ldots, k_{p}}^{\left(j_{m}\right)} \\
& = \begin{cases}(m !)^{-1} J_{l_{1}, \ldots, l_{p}}(f) & \text { if } l_{i}=i\left(j_{1}, \ldots, j_{m}\right):=\left|\left\{j_{u}=i: u=1, \ldots, m\right\}\right|, \\
0 & \text { otherwise. }\end{cases}
\end{aligned}
$$

At this point, we can close the proof since for $l_{i}=i\left(j_{1}, \ldots, j_{m}\right), 1 \leq i \leq p$, we have

$$
\tilde{J}_{j_{1}, \ldots, j_{m}}(f)=(m !)^{-1} J_{l_{1}, \ldots, l_{p}}(f) .
$$

\section{Acknowledgements}

This research was supported by Collaborative Research Center SFB 823 Statistical modeling of nonlinear dynamic processes. The author thanks two anonymous referees for their very detailed reviews on the submitted version of the paper. 


\section{References}

[1] Andrews, D.W.K. and Pollard, D. (1994). An introduction to functional central limit theorems for dependent stochastic processes. Int. Stat. Rev. 62 119-132.

[2] Arcones, M.A. (1994). Limit theorems for nonlinear functionals of a stationary Gaussian sequence of vectors. Ann. Probab. 22 2242-2274. MR1331224

[3] Bardet, J.-M. and Surgailis, D. (2013). Moment bounds and central limit theorems for Gaussian subordinated arrays. J. Multivariate Anal. 114 457-473. MR2993899

[4] Beran, J., Feng, Y., Ghosh, S. and Kulik, R. (2013). Long-Memory Processes. Heidelberg: Springer.

[5] Billingsley, P. (1968). Convergence of Probability Measures. New York-London-Sydney: Wiley. MR0233396

[6] Brockwell, P.J. and Davis, R.A. (1991). Time Series: Theory and Methods, 2nd ed. Springer Series in Statistics. New York: Springer.

[7] Dehling, H., Durieu, O. and Tusche, M. (2014). A sequential empirical CLT for multiple mixing processes with application to $\mathcal{B}$-geometrically ergodic Markov chains. Electron. J. Probab. 19 1-26.

[8] Dehling, H., Durieu, O. and Tusche, M. (2014). Approximating class approach for empirical processes of dependent sequences indexed by functions. Bernoulli 20 1372-1403. MR3217447

[9] Dehling, H. and Taqqu, M.S. (1989). The empirical process of some long-range dependent sequences with an application to $U$-statistics. Ann. Statist. 17 1767-1783. MR1026312

[10] Doukhan, P., Massart, P. and Rio, E. (1995). Invariance principles for absolutely regular empirical processes. Ann. Inst. Henri Poincaré B, Probab. Stat. 31 393-427. MR1324814

[11] Dudley, R.M. (1978). Central limit theorems for empirical measures. Ann. Probab. 6 899-929. MR0512411

[12] Fox, R. and Taqqu, M.S. (1987). Multiple stochastic integrals with dependent integrators. J. Multivariate Anal. 21 105-127. MR0877845

[13] Ho, H.C. and Sun, T.C. (1990). Limiting distributions of nonlinear vector functions of stationary Gaussian processes. Ann. Probab. 18 1159-1173. MR1062063

[14] Kechagias, S. and Pipiras, V. (2015). Definitions and representations of multivariate long-range dependent time series. J. Time Series Anal. 36 1-25.

[15] Leonenko, N.N., Sakhno, L. and Taufer, E. (2002). Product-limit estimator for long- and shortrange dependent sequences under gamma type subordination. Random Oper. Stoch. Equ. 10 301-320. MR1921856

[16] Marinucci, D. (2005). The empirical process for bivariate sequences with long memory. Stat. Inference Stoch. Process. 8 205-223.

[17] Ossiander, M. (1987). A central limit theorem under metric entropy with $L_{2}$ bracketing. Ann. Probab. 15 897-919. MR0893905

[18] Pipiras, V. and Taqqu, M.S. (2010). Regularization and integral representations of Hermite processes. Statist. Probab. Lett. 80 2014-2023. MR2734275

[19] Pollard, D. (1984). Convergence of Stochastic Processes. Springer Series in Statistics. New York: Springer. MR0762984

[20] Taqqu, M.S. (1975). Weak convergence to fractional Brownian motion and to the Rosenblatt process. Z. Wahrsch. Verw. Gebiete 31 287-302. MR0400329

[21] Taqqu, M.S. (1977). Law of the iterated logarithm for sums of non-linear functions of Gaussian variables that exhibit a long range dependence. Z. Wahrsch. Verw. Gebiete 40 203-238. MR0471045

[22] Taqqu, M.S. (1979). Convergence of integrated processes of arbitrary Hermite rank. Z. Wahrsch. Verw. Gebiete 50 53-83.

[23] Taufer, E. (2015). On the empirical process of strongly dependent stable random variables: Asymptotic properties, simulation and applications. Statist. Probab. Lett. 106 262-271. 
[24] van der Vaart, A.W. and Wellner, J.A. (1996). Weak Convergence and Empirical Processes: With Applications to Statistics. Springer Series in Statistics. New York: Springer. MR1385671

Received March 2016 and revised October 2016 